\title{
MANEJO DE PLANTAS DANINHAS NA CULTURA DE MILHO COM OS HERBICIDAS SULFENTRAZONE E CARFENTRAZONE-ETHYL
}

\author{
Pedro J. Christoffoleti', F. C. Machado², M. F. Amaral Oliveira ${ }^{2}$ e P.A. Monquero ${ }^{3}$
}

\footnotetext{
${ }^{1}$ Professor Associado. Departamento de Produção Vegetal, Escola Superior de Agricultura, Universidade de São Paulo (ESALQ/USP).

Caixa Postal 09. Piracicaba, SP 13418-900 pjchrist@esalq.usp.br

${ }^{2}$ Acadêmico de Agronomia. ESALQ/USP.

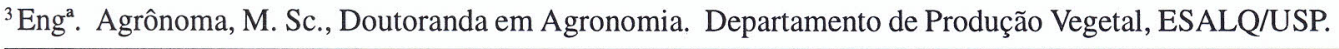

\section{RESUMO}

Dois experimentos foram desenvolvidos com o objetivo de avaliar o manejo de plantas daninhas na cultura de milho com o herbicida sulfentrazone aplicado em condições de pré-emergência, isolado ou em mistura com acetochlor, e do herbicida carfentrazone-ethyl aplicado em condições de pós-emergência, isolado ou em mistura com atrazine ou com nicosulfuron. Os experimentos foram conduzidos em delineamento experimental em blocos casualizados, com quatro repetições, no município de Piracicaba, SP, em área experimental do Departamento de Produção Vegetal da ESALQ/USP, durante o ano agrícola $2001 / 2002$. As avaliações efetuadas foram de percentagem de controle de plantas daninhas, efeitos tóxicos na cultura, produtividade de grãos e altura de plantas de milho. O herbicida sulfentrazone apresentou eficácia agronômica no controle de várias espécies de plantas daninhas em condições de pré-emergência na cultura de milho, quando aplicado nas doses de 0,15, 0,30 e 0,45 kg/ha isoladamente, ou nestas mesmas doses, em mistura com o herbicida acetochlor na dose de 2,52 kg/ha; o herbicida carfentrazoneethyl, em pós-emergência da cultura de milho, nas doses de 0,004 e $0,007 \mathrm{~kg} / \mathrm{ha}$ foi seletivo e eficiente no controle de plantas daninhas aplicado isoladamente, ou quando estas mesmas doses forem aplicadas em mistura com atrazine na dose de 2,5 kg/ ha e as plantas daninhas encontravam-se em média no estádio de pós-emergência de dois pares de folhas, ou quando estas doses de carfentrazone-ethyl foram aplicadas em mistura com o herbicida nicosulfuron na dose de $0,04 \mathrm{~kg} / \mathrm{ha}$ e as plantas daninhas encontravam-se em média no estádio de quatro pares de folhas.

Palavras-chaves: inibidores da PROTOX, seletividade.

\section{ABSTRACT \\ Weed management in corn with the herbicides sulfentrazone and carfentrazone-ethyl}

Experiments were carried out with the objective of evaluating the weed management in corn, spraying the herbicide sulfentrazone in pre-emergence conditions, alone or in combination with acetochlor and carfentrazone-ethyl herbicides in post-emergence conditions alone or in tank mix either with atrazine or nicosulfuron. The experiments were in completely randomized block design, with four replicates, in Piracicaba / SP, Brazil, in the experimental area of the Crop Production Department from University of São Paulo - ESALQ/USP. The weed control and the phytotoxic effects on the crop were evaluated as well as the final yield and plant height. The herbicide sulfentrazone was efficient in weed control and selective to corn crop sprayed in pre-emergence conditions at $0.15,0.30$ and $0.45 \mathrm{~kg} / \mathrm{ha}$ alone or when the same rates were applied in mixture with the herbicide acetochlor at $2.52 \mathrm{~kg} / \mathrm{ha}$; in post-emergence of the corn crop the herbicide carfentrazone-ethyl was selective and efficient in the control of weeds at the rates of $0.004 \mathrm{e} 0.007 \mathrm{~kg} / \mathrm{ha}$, sprayed alone or tank mix either with atrazine at $2.5 \mathrm{~kg} / \mathrm{ha}$, when the weeds are at the two pair of leaves growth stage; or with the herbicide nicosulfuron at the rate of 0.04 $\mathrm{kg} / \mathrm{ha}$, when the weeds are at the four pair of leaves stage.

Keywords: PROTOX inhibitors, selectivity. 
Pedro J. Christoffoleti et al.

\section{INTRODUÇÃO}

Herbicidas com bom desempenho biológico no controle de plantas daninhas são necessários para reduzir a interferência que as plantas daninhas causam à cultura do milho. A exigência cada vez maior de produtos mais seguros sob o ponto de vista ecológico e ambiental reforça ainda mais a necessidade de testes de novos produtos em desenvolvimento para o milho.

Carfentrazone-ethyl é um herbicida descoberto e patenteado pela FMC Corporation, sendo rapidamente absorvido pelas folhas (FMC Pesquisa. 1999 ${ }^{1}$ ). Seu mecanismo de ação está relacionado com a enzima protoporfirinogênio oxidase (PPO), responsável por uma das etapas de síntese da clorofila. A inibição da PPO resulta na formação de um oxigênio singlet, que promove a peroxidação lipídica e ruptura das membranas celulares, causando finalmente a morte da célula. A literatura científica registra um grande número de trabalhos que descrevem o mecanismo de ação dos herbicidas do grupo do carfentrazone (Kunert \& Dodge, 1989; Duke et al., 1991). O herbicida sulfentrazone age nas plantas por um processo de ruptura da membrana celular, sendo um herbicida sistêmico e de contato, absorvido primeiramente pelas raízes (Rodrigues $\&$ Almeida, 1995). Este herbicida tem grande potencial de utilização em pré-emergência em diversas culturas, destacandose, dentre estas, a soja e a cana-de-açúcar. No entanto, existem evidências de sua seletividade para outras culturas como milho e girassol.

A atuação da mistura de herbicidas pode ser sinergística, antagonística ou aditiva, quando comparada com a somatória das aplicações individuais de cada produto componente da mistura. Sinergismo ocorre quando o efeito observado da mistura é maior do que a resposta predita por um modelo estatístico apropriado, que considera o efeito dos produtos aplicados isoladamente. Antagonismo ocorre quando a aplicação conjunta dos herbicidas resulta em um controle menor do que o controle esperado pelo modelo. A interação aditiva ocorre quando o controle da planta daninha pela mistura é equivalente ao controle esperado (Colby, 1967). De um ponto de vista prático, antagonismo é a única resposta realmente indesejada, devido à diminuição da eficácia no controle das plantas daninhas e sinergismo é a interação desejada, pois aumenta o de controle das plantas daninhas (Starke \& Oliver, 1998). Muitos estudos documentam uma grande variedade de misturas de herbicidas que são sinergísticas (Lich et al., 1997; Blacksaw, 1989a; Blacksaw, 1989b; Riley \& Shaw, 1989; Minton et al., 1989; Hatzios, 1989; Riley \& Shaw, 1988; Sorenson et al., 1987). Estas misturas de herbicidas proporcionam um potencial para redução dos custos com herbicidas, bem como menor impacto ambiental (Harker \& O'Sullivan, 1991 e Jordan et al., 1997). Acrescenta-se a isso a possibilidade de controle de algumas espécies difíceis de serem controladas com doses de um herbicida único, aplicado convencionalmente.

${ }^{1}$ FMC pesquisa \& desenvolvimento. Carfentrazone-ethyl, produto em fase experimental. FMC, 18 p., 1999.
O objetivo principal desta pesquisa foi avaliar a praticabilidade de uso, eficácia de controle das plantas daninhas e seletividade para a cultura do milho do sulfentrazone aplicado em pré-emergência, isolado ou em mistura com acetochlor e do carfentrazone-ethyl aplicado em pós-emergência isolado, ou em mistura com atrazine ou nicosulfuron.

\section{MATERIAL E MÉTODOS}

Os experimentos foram conduzidos em área experimental pertencente ao Departamento de Produção Vegetal da Escola Superior de Agricultura "Luiz de Queiroz" Universidade de São Paulo (ESALQ-USP), Piracicaba, SP, em solo classificado como Latossolo vermelho eutrófico (Lve), com $45 \%$ de argila, $25 \%$ de silte e $30 \%$ de areia. O solo foi preparado através de uma aração e duas gradagens, sendo a segunda de nivelamento e efetuada dois dias antes da semeadura da cultura do milho, que ocorreu no dia 13/11/2001, utilizando o híbrido A 2288, de ciclo normal. Foi realizada uma adubação na semeadura com $400 \mathrm{~kg} / \mathrm{ha}$ do adubo formulado 08-28-16.

As parcelas experimentais mediam 3,6 $\mathrm{m}$ de largura (4 linhas espaçadas de $0,9 \mathrm{~m}$ entre si) x 5,0 m de comprimento, totalizando $18,0 \mathrm{~m}^{2}$ de área total, com quatro repetições. A área útil da parcela foi considerada como sendo as duas linhas centrais, eliminando $0,5 \mathrm{~m}$ em cada uma das extremidades.

Os herbicidas foram aplicados utilizando um pulverizador costal manual com pressão constante fornecida por $\mathrm{CO}_{2}$, e barra de aplicação provida de quatro bicos com pontas de pulverização do tipo 11003 , com consumo de calda de $200 \mathrm{~L} /$ ha e uma pressão de trabalho de $2,5 \mathrm{kgf} / \mathrm{cm}^{2}$

Para a análise estatística dos resultados foi feita inicialmente a análise de variância para obtenção dos valores $F$ para tratamentos; sendo este significativo procedeu-se o teste de Tukey para comparar as médias entre si. Ambos os testes foram feitos a $5 \%$ de probabilidade. Os testes de normalidade e homogeneidade dos dados de percentagem indicaram a necessidade de transformação antes de proceder análise, sendo então feita a transformação para arco seno da raiz quadrada da percentagem (percentagem de controle das plantas daninhas e índice de dessecação da parte aérea da cultura) dividido por 100. Os valores médios apresentados nas tabelas e figuras de resultados referem-se aos dados médios da percentagem de controle, sem transformação.

\section{Herbicida sulfentrazone}

Os tratamentos aplicados foram: sulfentrazone nas doses $0,15,0,30$ e $0,45 \mathrm{~kg} / \mathrm{ha}$, as mistura de sulfentrazone em cada uma destas doses mencionadas, com acetochlor na dose de $2,52 \mathrm{~kg} \mathrm{i.a/ha,} \mathrm{testemunha} \mathrm{capinada} \mathrm{e} \mathrm{testemunha} \mathrm{no} \mathrm{mato.}$ Os dados referentes à aplicação dos tratamentos foram: aplicação no dia 14/11/01, temperatura média do ar de $28^{\circ} \mathrm{C}$, umidade relativa do ar de $75 \%$, sem nuvens, velocidade do vento 
aproximadamente de $3 \mathrm{~m} /$ segundo, solo úmido na superfície, horário de aplicação das 9h25 (início) até as 10h (final). A primeira chuva (43 mm) ocorreu dois dias após a aplicação, sendo que a precipitação total ocorrida no período do experimento foi de $415 \mathrm{~mm}$.

As avaliações da percentagem de controle das plantas daninhas foram feitas através de uma escala percentual variando de 0 a 100 , onde zero significa ausência de controle e 100 controle total da plantas daninhas, aos 35 e 72 dias após aplicação dos herbicidas (DAT). Foram também avaliadas a produtividade final de milho em $\mathrm{kg} / \mathrm{ha}$ e altura das plantas de milho, em cm, medida aos 35 dias após o plantio.

As principais plantas daninhas existentes na área experimental, cuja densidade mínima era de 10 plantas $/ \mathrm{m}^{2} \mathrm{eram}$ : Alternanthera tenella (apaga-fogo), Brachiaria plantaginea (capim-marmelada), Desmodium tortuosum (carrapicho-beiçode-boi), Acanthospermum hispidum (carrapicho-rasteiro), Bidens pilosa (picão-preto), Emilia sonchifolia (falsaserralha), Portulaca oleracea (beldroega), Blainvillea rhomboidea (erva-palha), Indigofera hirsuta (anileira), Richardia brasiliensis (poaia-branca), Commelina benghalensis (trapoeraba) e Oxalis sp (trevo).

\section{Herbicida carfentrazone-ethyl}

Os tratamentos utilizados foram: carfentrazone-ethyl nas doses 0,004 e $0,007 \mathrm{~kg} / \mathrm{ha}$, aplicado isoladamente em cada uma destas doses em mistura com atrazine $(2,5 \mathrm{~kg} / \mathrm{ha}) \mathrm{e}$ nicosulfuron $(0,04 \mathrm{~kg} / \mathrm{ha})$, testemunha capinada e testemunha no mato. Os dados referentes à aplicação dos tratamentos foram os seguintes: temperatura média do ar de $26^{\circ} \mathrm{C}$, umidade relativa do ar de $60 \%$, condições do tempo (nebulosidade do céu) sem nuvens, velocidade do vento aproximadamente de 3 $\mathrm{m} / \mathrm{segundo}$, solo úmido na superfície, horário de aplicação das $8 \mathrm{~h}$ (início) até as $9 \mathrm{~h} 10$ (final). A primeira chuva (38 mm) ocorreu sete dias após a aplicação, sendo que a precipitação total ocorrida no período do experimento foi de $415 \mathrm{~mm}$.

As principais plantas daninhas existentes na área experimental eram: Alternanthera tenella (apaga-fogo), Brachiaria plantaginea (capim-marmelada), Desmodium tortuosum (carrapicho-beiço-de-boi), Acanthospermum hispidum (carrapicho-rasteiro), Portulaca oleracea (beldroega), Indigofera hirsuta (anileira), Richardia brasiliensis (poaia-branca), Commelina benghalensis (trapoeraba) e Sida rhombifolia (guanxuma).

Os tratamentos com o herbicida carfentrazone-ethyl isolado ou em mistura com atrazine foram aplicados com as plantas daninhas no estádio médio de quatro folhas verdadeiras e os tratamentos com a mistura dos herbicidas carfentrazone-ethyl + nicosulfuron foram aplicados no estádio de oito folhas verdadeiras.

As avaliações da percentagem de controle das plantas daninhas foram feitas através de uma escala percentual variando de 0 a 100 , onde zero significa ausência de controle e 100 controle total da plantas daninhas, aos 35, 60 e 72 dias após aplicação dos herbicidas (DAT). A primeira avaliação não foi analisada estatisticamente, pois apenas dois tratamentos haviam sido aplicados até o momento.

Foram também avaliados possíveis sintomas visuais de fitotoxicidade onde zero significava ausência de efeito tóxico e 9 a morte total da planta, aos 35, 60 e 75 dias após a semeadura. $\mathrm{Na}$ avaliação de fitotoxicidade aos 35 dias após a semeadura foram avaliados apenas os tratamentos 1 e 2 pois os demais tratamentos não haviam sido pulverizados até esta avaliação. A produtividade final de milho em $\mathrm{kg} / \mathrm{ha}$ e altura das plantas de milho em $\mathrm{cm}$ medida aos 45 dias após o plantio, também foram analisadas.

\section{RESULTADOS E DISCUSSÃO}

Controle de plantas daninhas com o herbicida sulfentrazone

Todos os tratamentos herbicidas foram eficientes no controle geral das plantas daninhas, pois estatisticamente todos estes tratamentos foram semelhantes à testemunha capinada, a qual foi mantida livre da competição de plantas daninhas durante todo o ciclo (Figua 1). Apenas o tratamento que foi mantido em competição com o mato durante todo o ciclo da cultura diferiu estatisticamente dos demais, indicando que a infestação de plantas daninhas existentes nas parcelas era suficiente para interferir significativamente na produção da cultura. De acordo com Almeida (1981), a redução no rendimento desta cultura devido à competição estabelecida com as plantas daninhas pode variar de 12 a $100 \%$, em função da espécie, grau de infestação, tipo de solo, condições climáticas, além do estádio fenológico da cultura. Também observase que a altura das plantas de milho aos 35 DAT era semelhante em todos os tratamentos com herbicidas e na testemunha capinada; porém na testemunha mantida no mato era maior (Figura 1). Este fato deveu-se à competição pela luz ocorrida nesta parcela durante os primeiros 35 dias, pois a resposta da planta à competição pela luz é o estiolamento (Zimdhal, 1990). Outros pesquisadores constatam que a partir da emissão da quinta folha, a presença de plantas daninhas na cultura do milho reduz o rendimento de grãos, comprimento médio da espiga e número médio de grãos por fileira (Fancelli \& Dourado Neto, 2000).

As plantas daninhas que foram controladas satisfatoriamente (controle entre $90-100 \%$ ) nas duas avaliações (até 72 dias após aplicação dos herbicidas) foram Alternanthera tenella, Brachiaria plantaginea, Desmodium tortuosum, Acanthospermum hispidum,Bidens pilosa, Emilia sonchifolia, Portulaca oleracea, e Blainvillea rhomboidea. A planta daninha Indigofera hirsuta apenas não foi controlada na menor dose do herbicida sulfentrazone $(0,15 \mathrm{~kg} / \mathrm{ha})$ aos 72 DAT; no entanto, nos demais tratamentos foi satisfatoriamente controlada. A planta daninha Richardia brasiliensis foi controlada em mais de $90 \%$ apenas na dose mais elevada de sulfentrazone $(0,45 \mathrm{~kg} / \mathrm{ha})$ quando aplicado isoladamente, porém quando o sulfentrazone foi misturado com o herbicida acetochlor todas as doses testadas controlaram satisfatoriamente a poaia-bran- 
Pedro J. Christoffoleti et al.

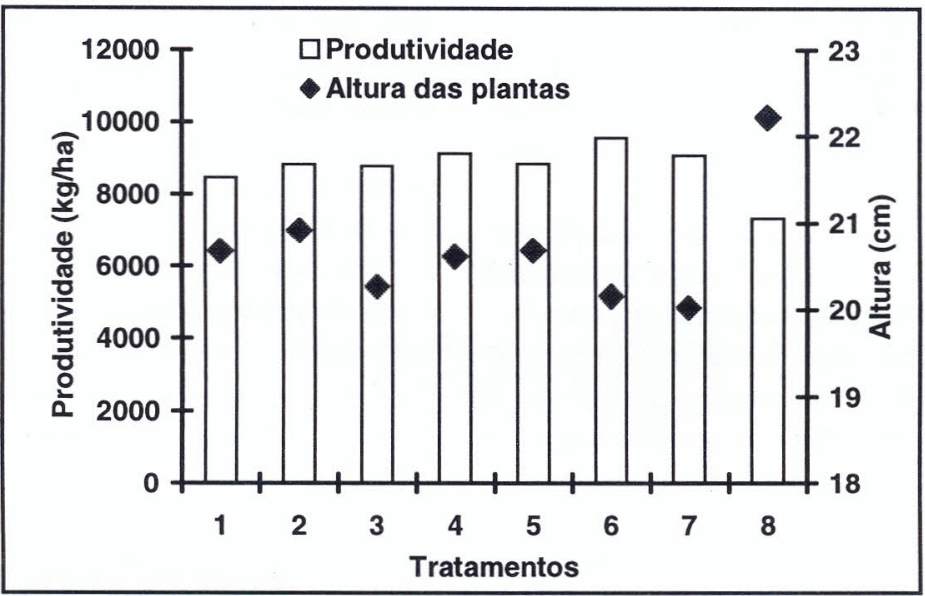

\begin{tabular}{|c|c|c|}
\hline Tratamento (kg/ha) & Produtividade (kg/ha) & Altura das plantas (cm) \\
\hline 1. Sulfentrazone $(0,15)$ & $8469 a^{1}$ & $20,7 b$ \\
\hline 2. Sulfentrazone $(0,30)$ & $8843 a$ & $20,9 b$ \\
\hline 3. Sulfentrazone $(0,45)$ & $8773 a$ & $20,3 b$ \\
\hline 4. Sulfentrazone $(0,15)+$ acetochlor $(2,52)$ & $9123 a$ & $20,6 b$ \\
\hline 5. Sulfentrazone $(0,30)+$ acetochlor $(2,52)$ & $8854 a$ & $20,7 b$ \\
\hline 6. Sulfentrazone $(0,45)+$ acetochlor $(2,52)$ & $9551 a$ & $20,2 b$ \\
\hline 7. Testemunha capinada & $9066 \mathrm{a}$ & $20,0 \mathrm{~b}$ \\
\hline 8. Testemunha no mato & $7332 b$ & $22,2 \mathrm{a}$ \\
\hline $\mathrm{F}(5 \%)$ & $* *$ & $* *$ \\
\hline C.V. $(\%)$ & 12,4 & 9,7 \\
\hline
\end{tabular}

Figura 1. Efeito do herbicida sulfentrazone aplicado isolado ou em mistura, em pré-emergência, sobre a produtividade e altura das plantas de milho (medida 35 dias após plantio). Médias acompanhadas de mesma letra não diferem estatisticamente entre si através do teste de Tukey ao nível de $1 \%$ de probabilidade. ** teste $\mathrm{F}$ significativo ao nível de $1 \%$ de probabilidade.

ca. Por sua vez, Monquero et al., (2001) observaram que o herbicida sulfentrazone $(0,70 \mathrm{~kg} / \mathrm{ha})$ aplicado isoladamente e em mistura com glyphosate em pós-emergência, não controla satisfatoriamente esta espécie, sendo que a interação de glyphosate + sulfentrazone é antagônica.

A planta daninha Commelina benghalensis foi controlada adequadamente por todos os tratamentos, exceto pela menor dose do sulfentrazone isoladamente. Nenhum tratamento controlou adequadamente Oxalis sp, porém este resultado era esperado, pois esta planta daninha apresenta propagação vegetativa por bulbos subterrâneos que não são controlados pelos herbicidas utilizados (Tabela 1).

Nenhum tratamento herbicida utilizado no experimento evidenciou sintomas visuais de fitotoxicidade até os 72 DAT, portanto, os resultados não foram apresentados. Desta forma, o herbicida sulfentrazone, nas doses $0,15,0,30$ e $0,45 \mathrm{~kg} / \mathrm{ha}$, aplicado isoladamente ou em mistura com o herbicida acetochlor foi seletivo para a cultura do milho quando aplicado em condições de pré-emergência da cultura e das plantas daninhas. O herbicida sulfentrazone, nas doses estudadas e em misturas com acetochlor, controlou um grande número de espécies de plantas daninhas e apresentou seletividade para a cultura do milho.

Controle de plantas daninhas com o herbicida carfentrazoneethyl

O herbicida carfentrazone-ethyl é registrado no Brasil para as culturas de milho, soja, algodão, café e citros controlando, principalmente, plantas daninhas dicotiledôneas.

Todos os tratamentos que receberam tratamentos herbicidas produziram quantidades semelhantes de grãos, do mesmo modo que a testemunha capinada, onde não houve competição com as plantas daninhas (Figura 2). No tratamento mantido em total competição com as plantas daninhas (testemunha no mato), houve menor produtividade, devido à intensa competição. Sendo assim, todos os tratamentos herbicidas controlaram de forma geral todas as plantas daninhas incidentes no ensaio. Os resultados da altura de plantas de milho também demonstram que a testemunha mantida no mato apresentou estiolamento das plantas de milho devido à competição pela luz. 
Manejo de plantas daninhas na cultura de milho com os herbicidas sulfentrazone e carfentrazone-ethyl

Tabela 1. Percentagem de controle das plantas daninhas com o herbicida sulfentrazone aplicado em condições de pré-emergência, aos 35 e 72 DAT. Piracicaba, 2001/2002. Médias acompanhadas de mesma letra não diferem estatisticamente entre si pelo teste de Tukey a $1 \%$ de probabilidade. ** teste $\mathrm{F}$ significativo ao nível de $1 \%$ de probabilidade.

\begin{tabular}{|c|c|c|c|c|c|c|c|c|}
\hline \multirow[t]{2}{*}{ Tratamento (kg/ha) } & \multicolumn{2}{|c|}{ INDHI } & \multicolumn{2}{|c|}{ ALRTE } & \multicolumn{2}{|c|}{ BRAPL } & \multicolumn{2}{|c|}{ DEDTO } \\
\hline & 35 & 72 & 35 & 72 & 35 & 72 & 35 & 72 \\
\hline 1. Sulfentrazone $(0,15)$ & 100 & $62 b$ & 100 & 100 & 100 & 100 & 100 & $100 \mathrm{a}$ \\
\hline 2. Sulfentrazone $(0,30)$ & 100 & $95 a$ & 100 & 100 & 100 & 100 & 100 & $97,5 \mathrm{a}$ \\
\hline 3. Sulfentrazone $(0,45)$ & 100 & $97 \mathrm{a}$ & 100 & 100 & 100 & 100 & 100 & $100 \mathrm{a}$ \\
\hline 4. Sulfent. $(0,15)+$ Acet. $(2,52)$ & 100 & $100 \mathrm{a}$ & 100 & 100 & 100 & 100 & 100 & $100 \mathrm{a}$ \\
\hline 5. Sulfent. $(0,30)+$ Acet. $(2,52)$ & 100 & $100 \mathrm{a}$ & 100 & 100 & 100 & 100 & 100 & $100 \mathrm{a}$ \\
\hline 6. Sulfent. $(0,45)+$ Acet. $(2,52)$ & 100 & $100 \mathrm{a}$ & 100 & 100 & 100 & 100 & 100 & $100 \mathrm{a}$ \\
\hline 7. Testemunha capinada & 100 & $100 \mathrm{a}$ & 100 & 100 & 100 & 100 & 100 & $100 \mathrm{a}$ \\
\hline 8. Testemunha no mato & 0 & 0 & 0 & 0 & 0 & 0 & 0 & $\mathrm{Ob}$ \\
\hline $\mathrm{F}(5 \%)$ & - & $* *$ & - & - & - & - & - & $* *$ \\
\hline C.V. (\%) & - & 13,2 & - & - & - & - & - & 14,6 \\
\hline \multirow[t]{2}{*}{ Tratamento } & \multicolumn{2}{|c|}{ ACNHI } & \multicolumn{2}{|c|}{ RICHIBR } & \multicolumn{2}{|c|}{ COMBE } & \multicolumn{2}{|c|}{ OXALA } \\
\hline & 35 & 72 & 35 & 72 & 35 & 72 & 35 & 72 \\
\hline 1. Sulfentrazone $(0,15)$ & 100 & $95 a$ & $84 b$ & $87 \mathrm{~b}$ & $71 \mathrm{~b}$ & $96 a$ & $80 \mathrm{~b}$ & $85 \mathrm{ab}$ \\
\hline 2. Sulfentrazone $(0,30)$ & 100 & $100 \mathrm{a}$ & $90 \mathrm{ab}$ & $85 b$ & $99 a$ & $97 a$ & $78 b$ & $66 b$ \\
\hline 3. Sulfentrazone $(0,45)$ & 100 & $100 \mathrm{a}$ & $95 \mathrm{ab}$ & $91 \mathrm{a}$ & $100 \mathrm{a}$ & $100 \mathrm{a}$ & $88 \mathrm{a}$ & $62 b$ \\
\hline 4. Sulfent. $(0,15)+$ Acet. $(2,52)$ & 100 & $100 \mathrm{a}$ & $100 \mathrm{a}$ & $99 \mathrm{a}$ & $100 \mathrm{a}$ & $95 \mathrm{a}$ & $97 \mathrm{a}$ & $80 \mathrm{~b}$ \\
\hline 5. Sulfent. $(0,30)+$ Acet. $(2,52)$ & 100 & $100 \mathrm{a}$ & $100 \mathrm{a}$ & $100 \mathrm{a}$ & $100 \mathrm{a}$ & $100 \mathrm{a}$ & $92 \mathrm{a}$ & $76 b$ \\
\hline 6. Sulfent. $(0,45)+$ Acet. $(2,52)$ & 100 & $100 \mathrm{a}$ & $100 \mathrm{a}$ & $100 \mathrm{a}$ & $100 \mathrm{a}$ & $97 \mathrm{a}$ & $77 \mathrm{~b}$ & $67 b$ \\
\hline 7. Testemunha capinada & 100 & $100 \mathrm{a}$ & $100 \mathrm{a}$ & $100 \mathrm{a}$ & $100 \mathrm{a}$ & $100 \mathrm{a}$ & $100 \mathrm{a}$ & $100 \mathrm{a}$ \\
\hline 8. Testemunha no mato & 0 & $\mathrm{Ob}$ & $0 \mathrm{c}$ & $0 \mathrm{c}$ & $0 \mathrm{c}$ & $\mathrm{Ob}$ & $0 \mathrm{c}$ & $0 \mathrm{c}$ \\
\hline $\mathbf{F}(5 \%)$ & - & $* *$ & $* *$ & $* *$ & $* *$ & $* *$ & $* *$ & $* *$ \\
\hline C.V. $(\%)$ & - & 12,8 & 13,2 & 16,3 & 13,9 & 16,8 & 15,5 & 12,2 \\
\hline \multirow[t]{2}{*}{ Tratamento } & \multicolumn{2}{|c|}{ BIDPI } & \multicolumn{2}{|c|}{ EMISO } & \multicolumn{2}{|c|}{ POROL } & \multicolumn{2}{|c|}{ BLARH } \\
\hline & 35 & 72 & 35 & 72 & 35 & 72 & 35 & 72 \\
\hline 1. Sulfentrazone $(0,15)$ & 100 & 100 & 100 & 100 & 100 & 100 & 100 & 100 \\
\hline 2. Sulfentrazone $(0,30)$ & 100 & 100 & 100 & 100 & 100 & 100 & 100 & 100 \\
\hline 3. Sulfentrazone $(0,45)$ & 100 & 100 & 100 & 100 & 100 & 100 & 100 & 100 \\
\hline 4. Sulfent. $(0,15)+$ Acet. $(2,52)$ & 100 & 100 & 100 & 100 & 100 & 100 & 100 & 100 \\
\hline 5. Sulfent. $(0,30)+$ Acet. $(2,52)$ & 100 & 100 & 100 & 100 & 100 & 100 & 100 & 100 \\
\hline 6. Sulfent. $(0,45)+$ Acet. $(2,52)$ & 100 & 100 & 100 & 100 & 100 & 100 & 100 & 100 \\
\hline 7. Testemunha capinada & 100 & 100 & 100 & 100 & 100 & 100 & 100 & 100 \\
\hline 8. Testemunha no mato & 0,0 & 0,0 & 0,0 & 0,0 & 0,0 & 0,0 & 0,0 & 0,0 \\
\hline $\mathrm{F}(5 \%)$ & - & - & - & - & - & - & - & - \\
\hline C.V. $(\%)$ & - & - & - & - & - & 一 & - & - \\
\hline
\end{tabular}

Códigos das plantas daninhas = nome científico (nome comum): $\mathbf{I N D H I}=$ Indigofera hirsuta $($ anileira $) ; \mathbf{A L R T E}=$ Alternanthera tenella (apaga-fogo); BRAPL = Brachiaria plantaginea (capim-marmelada); DEDTO = Desmodium tortuosum (carrapicho-beiço-de-boi); ACNHI $=$ Acanthospermum hispidum (carrapicho-rasteiro); $\mathbf{R I C H B R}=$ Richardia brasiliensis (poaia-branca); $\mathbf{C O M B E}=$ Commelina benghalensis (trapoeraba); OXALA = Oxalis sp; BIDPI = Bidens pilosa (picão-preto); EMISO = Emilia sonchifolia (falsa-serralha); POROL = Portulaca oleracea (beldroega); BLARH = Blainvillea rhomboidea (erva-palha). 
Pedro J. Christoffoleti et al.

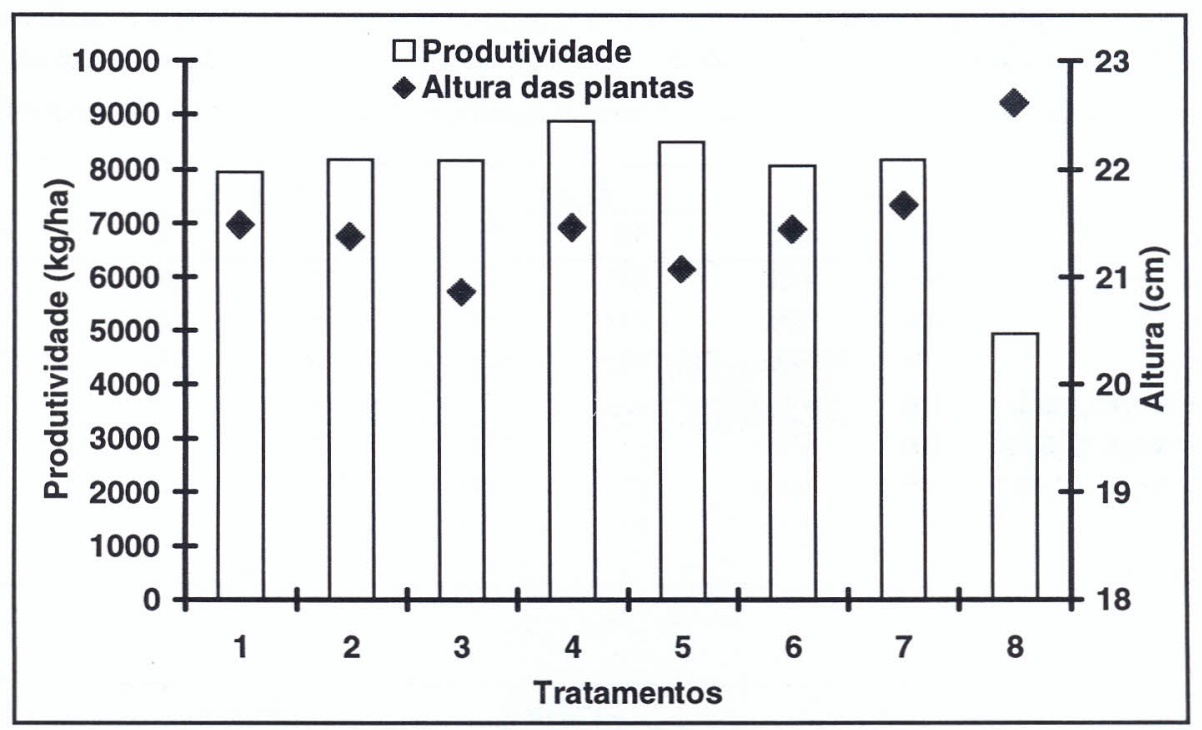

\begin{tabular}{|c|c|c|}
\hline Tratamento (kg/ha) & Produtividade (kg/ha) & Altura das plantas (cm) \\
\hline 1. Carfentrazone $(0,004)+$ atrazine $(2,5)$ & $7947 \mathrm{a}$ & $21,5 b$ \\
\hline 2. Carfentrazone $(0,007)+$ atrazine $(2,5)$ & $8179 a$ & $21,4 b$ \\
\hline 3. Carfentrazone $(0,004)+$ nicosulfuron $(0,04)$ & $8152 \mathrm{a}$ & $20,9 b$ \\
\hline 4. Carfentrazone $(0,007)+$ nicosulfuron $(0,04)$ & $8888 \mathrm{a}$ & $21,5 b$ \\
\hline 5.Carfentrazone $(0,004)$ & $8497 a$ & $21,1 b$ \\
\hline 6.Carfentrazone $(0,007)$ & $8066 \mathrm{a}$ & $21,5 b$ \\
\hline 7.Testemunha capinada & $817 \mathrm{a}$ & $21,7 b$ \\
\hline 8.Testemunha no mato & $4944 b$ & $22,6 \mathrm{a}$ \\
\hline $\mathrm{F}(\mathbf{5 \%})$ & $* *$ & $* *$ \\
\hline C.V. $(\%)$ & 16,7 & 13,7 \\
\hline
\end{tabular}

Figura 2. Efeito do herbicida carfentrazone-ethyl aplicado isolado ou em mistura, em pré-emergência, sobre a produtividade de milho e altura das plantas de milho (medida aos 45 dias após a semeadura). Médias acompanhadas de mesma letra não diferem estatisticamente entre si através do teste de Tukey ao nível de $1 \%$ de probabilidade. ** teste F significativo ao nível de $1 \%$ de probabilidade.

A aplicação de herbicidas em pós-emergência inicial e em pós-emergência dirigida pode causar injúrias às plantas de milho, quando estas são atingidas por produtos não totalmente seletivos. Os resultados de avaliação visual de fitotoxicidade mostram que a mistura do herbicida carfentrazone $(0,018 \mathrm{~kg} / \mathrm{ha})+$ nicosulfuron $(0,04 \mathrm{~kg} / \mathrm{ha})$ foi a que causou maiores sintomas de fitotoxicidade; porém, em níveis considerados aceitáveis, ou seja, no máximo 2,0 (Tabela 2). Não foram observados sintomas visuais de fitotoxicidade após os 75 dias da semeadura da cultura de milho. Portanto, o herbicida carfentrazone, nas doses de 0,010 e $0,018 \mathrm{~kg} / \mathrm{ha}$, isolado ou em mistura com nicosulfuron e atrazine foi seletivo para a cultura de milho, quando aplicado em condições de pós-emergência.

A planta daninha anileira (Indigofera hirsuta) foi satisfatoriamente controlada pela mistura dos herbicidas carfentrazone e atrazine nas duas doses estudadas (Tabela 3). Quando em mistura com nicosulfuron o controle não foi total, porém superior a $80 \%$ na avaliação de 75 DAA. Quando o herbicida carfentrazone foi aplicado isoladamente não houve controle satisfatório da planta daninha. O capim-marmelada só não foi controlado pelo tratamento com a mistura de carfentrazone + atrazine. Segundo Rodrigues et al., (2000), atrazine em mistura com os dessecantes glyphosate, paraquat e 2-4D são eficientes no controle desta espécie.

Desmodium tortuosum (carrapicho-beiço-de-boi) não foi controlado pelo carfentrazone-ethyl isoladamente; porém, quando em mistura com atrazine ou nicosulfuron o controle foi excelente (Tabela 3). O apaga-fogo (Althernanthera tenella) foi controlado satisfatoriamente por todos os tratamentos; porém, quando o carfentrazone-ethyl foi aplicado em mistura com atrazine ou nicosulfuron houve efeito aditivo destes herbicidas. As plantas daninhas Acanthospermum hispidum (carrapicho-rasteiro); Richardia brasiliensis (poaiabranca), Sida rhombifolia (guanxuma) e Portulaca oleracea (beldroega) foram todas controladas pelo herbicida carfentrazone em mistura com nicosulfuron ou atrazine, porém, não apresentou controle satisfatório quando aplicado sozinho. Commelina benghalensis (trapoeraba) foi controla satisfatoriamente pelo carfentrazone-ethyl, mesmo quando 
Tabela 2. Fitotoxicidade média observada nos tratamentos do experimento com o herbicida carfentrazone-ethyl aplicado em condições de pós-emergência na cultura do milho aos 35, 60 e 75 dias após a semeadura da cultura. Piracicaba, 2001/2002.

\begin{tabular}{|c|c|c|c|}
\hline \multirow[t]{2}{*}{ Tratamento (kg/ha) } & \multicolumn{3}{|c|}{ Dias após a semeadura da cultura } \\
\hline & 35 & 60 & 75 \\
\hline 1.Carfentrazone $(0,004)+$ atrazine $(2,5)$ & 1,3 & 0,5 & 0,0 \\
\hline 2. Carfentrazone $(0,007)+$ atrazine $(2,5)$ & 1,8 & 1,0 & 0,0 \\
\hline 3.Carfentrazone $(0,004)+$ nicosulfuron $(0,04)$ & - & 1,5 & 0,0 \\
\hline 4.Carfentrazone $(0,007)+$ nicosulfuron $(0,04)$ & - & 2,0 & 0,8 \\
\hline 5.Carfentrazone $(0,004)$ & - & 1,0 & 0,3 \\
\hline 6.Carfentrazone $(0,007)$ & - & 1,5 & 0,3 \\
\hline 7.Testemunha capinada & - & - & - \\
\hline 8.Testemunha no mato & - & - & - \\
\hline
\end{tabular}

Observações: Os resultados destas avaliações não foram analisados estatisticamente devido à baixa variabilidade dos mesmos. Após os 75 dias após o plantio não foram mais observados sintomas visuais de fitotoxicidade.

Tabela 3. Porcentagem de controle das plantas daninhas através do herbicida carfentrazone-ethyl. Piracicaba, $2001 / 2002$. Médias acompanhadas de mesma letra não diferem estatisticamente entre si através do teste de Tukey ao nível de $1 \%$ de probabilidade. ** teste $\mathrm{F}$ significativo ao nível de $1 \%$ de probabilidade.

\begin{tabular}{|c|c|c|c|c|c|c|c|c|c|}
\hline \multirow[t]{2}{*}{ Tratamento } & \multicolumn{3}{|c|}{ INDHI } & \multicolumn{3}{|c|}{ BRAPL } & \multicolumn{3}{|c|}{ DEDTO } \\
\hline & 35 & 60 & 75 & 35 & 60 & 75 & 35 & 60 & 75 \\
\hline 1.Carfent. $(0,004)+$ Atraz. $(2,5)$ & 100 & $75 b$ & $100 \mathrm{a}$ & 37 & $25 \mathrm{~d}$ & $37,5 c$ & 100 & $75 c$ & $100 \mathrm{a}$ \\
\hline 2.Carfent. $(0,007)+$ Atraz. $(2,5)$ & 100 & $75 b$ & $97 \mathrm{a}$ & 87 & $75 c$ & $80,0 \mathrm{~b}$ & 100 & $100 \mathrm{a}$ & $100 \mathrm{a}$ \\
\hline 3.Carfent. $(0,004)+$ Nicos. $(0,04)$ & - & $57 \mathrm{c}$ & $86 a$ & -- & $82 b$ & $81,3 b$ & -- & $92 b$ & $90 \mathrm{a}$ \\
\hline 4.Carfent. $(0,007)+$ Nicos. $(0,04)$ & - & $55 \mathrm{c}$ & $85 \mathrm{a}$ & -- & $90 \mathrm{ab}$ & $87,5 b$ & - & $91 b$ & $92 a$ \\
\hline 5.Carfentrazone $(0,004)$ & - & $26 \mathrm{~d}$ & $35 b$ & - & $75 c$ & $80,0 \mathrm{~b}$ & - & $0 \mathrm{e}$ & $10 \mathrm{c}$ \\
\hline 6.Carfentrazone $(0,007)$ & - & $45 \mathrm{c}$ & $50 \mathrm{~b}$ & -- & $75 c$ & $81,3 b$ & - & $50 \mathrm{c}$ & $47 b$ \\
\hline 7.Testemunha capinada & 100 & $100 \mathrm{a}$ & $100 \mathrm{a}$ & 100 & $100 \mathrm{a}$ & $100 \mathrm{a}$ & 100 & $100 \mathrm{a}$ & $100 \mathrm{a}$ \\
\hline 8.Testemunha no mato & 0 & 0e & $0 \mathrm{c}$ & 0 & $0 \mathrm{e}$ & $0,0 \mathrm{~d}$ & 0 & $0 \mathrm{e}$ & $0 \mathrm{~d}$ \\
\hline F (5\%) & -- & $* *$ & $* *$ & - & $* *$ & $* *$ & -- & $* *$ & $* *$ \\
\hline C.V. & -- & 23,7 & 16,9 & - & 16,8 & 22,9 & - & 17,5 & 20,0 \\
\hline \multirow[t]{2}{*}{ Tratamento } & \multicolumn{3}{|c|}{ ALRTE } & \multicolumn{3}{|c|}{ ACNHI } & \multicolumn{3}{|c|}{ RICHBR } \\
\hline & 35 & 60 & 75 & 35 & 60 & 75 & 35 & 60 & 75 \\
\hline 1.Carfent. 0,004 + Atraz. 2,5 & 100 & $100 \mathrm{a}$ & $100 \mathrm{a}$ & 100 & $100 \mathrm{a}$ & $100 \mathrm{a}$ & 100 & $100 \mathrm{a}$ & $100 \mathrm{a}$ \\
\hline 2.Carfent. 0,007+ Atraz. 2,5 & 100 & $100 \mathrm{a}$ & $100 \mathrm{a}$ & 100 & $100 \mathrm{a}$ & $100 \mathrm{a}$ & 100 & $100 \mathrm{a}$ & $100 \mathrm{a}$ \\
\hline 3.Carfent. 0,004 + Nicos. 0,04 & -- & $100 \mathrm{a}$ & $100 \mathrm{a}$ & - & $100 \mathrm{a}$ & $100 \mathrm{a}$ & - & $81 b$ & $81 b$ \\
\hline 4.Carfent. 0,007 + Nicos. 0,04 & - & $100 \mathrm{a}$ & $100 \mathrm{a}$ & - & $92 \mathrm{a}$ & $97 \mathrm{a}$ & - & $86 b$ & $84 b$ \\
\hline 5.Carfentrazone 0,004 & - & $80 \mathrm{~b}$ & $85 b$ & - & $5 c$ & $17 \mathrm{c}$ & - & $5 c$ & $42 \mathrm{c}$ \\
\hline 6.Carfentrazone 0,007 & -- & $86 b$ & $89 b$ & - & $42 b$ & $42 b$ & - & $14 \mathrm{c}$ & $25 \mathrm{~d}$ \\
\hline 7.Testemunha capinada & 100 & $100 \mathrm{a}$ & $100 \mathrm{a}$ & 100 & $100 \mathrm{a}$ & $100 \mathrm{a}$ & 100 & $100 \mathrm{a}$ & $100 \mathrm{a}$ \\
\hline 8.Testemunha no mato & 0 & $0 \mathrm{~d}$ & $0 \mathrm{c}$ & 0 & $0 \mathrm{c}$ & $0 \mathrm{~d}$ & 0 & $0 \mathrm{c}$ & $0 \mathrm{e}$ \\
\hline F (5\%) & - & $* *$ & $* *$ & - & $* *$ & $* *$ & - & $* *$ & $* *$ \\
\hline C.V. & -- & 12,5 & 14,4 & 一- & 17,4 & 18,0 & 一 & 13,4 & 15,9 \\
\hline \multirow[t]{2}{*}{ Tratamento } & \multicolumn{3}{|c|}{ COMBE } & \multicolumn{3}{|c|}{ SIDRH } & \multicolumn{3}{|c|}{ POROL } \\
\hline & 35 & 60 & 75 & 35 & 60 & 75 & 35 & 60 & 75 \\
\hline 1.Carfent. 0,004 + Atraz. 2,5 & 100 & $96 a$ & $94 a$ & 100 & $100 \mathrm{a}$ & $100 \mathrm{a}$ & 100 & $100 \mathrm{a}$ & $100 \mathrm{a}$ \\
\hline 2.Carfent. 0,007+ Atraz. 2,5 & 100 & $97 a$ & $93 \mathrm{a}$ & 100 & $100 \mathrm{a}$ & $100 \mathrm{a}$ & 100 & $100 \mathrm{a}$ & $100 \mathrm{a}$ \\
\hline 3.Carfent. 0,004 + Nicos. 0,04 & - & $93 a$ & $90 \mathrm{a}$ & -- & $82 b$ & $77 b$ & - & $78 b$ & $80 \mathrm{~b}$ \\
\hline 4.Carfent. 0,007 + Nicos. 0,04 & - & $94 \mathrm{ab}$ & $90 \mathrm{a}$ & - & $75 \mathrm{c}$ & $80 b$ & - & $100 \mathrm{a}$ & $98 \mathrm{a}$ \\
\hline 5.Carfentrazone 0,004 & - & $80 \mathrm{~b}$ & $82 b$ & -- & $70 \mathrm{c}$ & $75 b$ & - & $20 \mathrm{~d}$ & $20 \mathrm{~d}$ \\
\hline 6.Carfentrazone 0,007 & - & $92 \mathrm{ab}$ & $91 \mathrm{a}$ & - & $45 \mathrm{~d}$ & $51 \mathrm{c}$ & - & $50 \mathrm{c}$ & $60 \mathrm{c}$ \\
\hline 7.Testemunha capinada & & $100 \mathrm{a}$ & $100 \mathrm{a}$ & 100 & $100 \mathrm{a}$ & $100 \mathrm{a}$ & 100 & $100 \mathrm{a}$ & $100 \mathrm{a}$ \\
\hline 8. Testemunha no mato & 0 & $0 \mathrm{c}$ & $0 \mathrm{c}$ & 0 & $0 \mathrm{e}$ & $0 \mathrm{e}$ & 0 & $0 \mathrm{e}$ & $0 \mathrm{e}$ \\
\hline F (5\%) & - & $* *$ & $* *$ & - & $* *$ & $* *$ & - & $* *$ & $* *$ \\
\hline C.V. & - & 14,8 & 13,8 & -- & 15,8 & 15,0 & - & 16,6 & 14,3 \\
\hline
\end{tabular}

Códigos das plantas daninhas $=$ nome científico $($ nome comum): $\mathbf{I N D H I}=$ Indigofera hirsuta $($ anileira $) ;$ BRAPL $=$ Brachiaria plantaginea (capim-marmelada); DEDTO = Desmodium tortuosum (carrapicho-beiço-de-boi); ALRTE = Alternanthera tenella $($ apaga-fogo); $\mathbf{A C N H I}=$ Acanthospermum hispidum (carrapicho-rasteiro); RICHBR = Richardia brasiliensis (poaia-branca); COMBE = Commelina benghalensis (trapoeraba); SIDRH = Sida rhombifolia $($ guanxuma); POROL $=$ Portulaca oleracea (beldroega). 
Pedro J. Christoffoleti et al.

aplicado isoladamente nas duas doses estudadas. Monquero et al., (2001) observaram o controle satisfatório de $C$. benghalensis, $R$. brasiliensis, I grandifolia, A. hybridus e Galinsoga parviflora pelos herbicidas carfentrazone-ethyl $(0,03 \mathrm{~kg} / \mathrm{ha})$ e flumioxazin $(0,05 \mathrm{~kg} / \mathrm{ha})$ aplicados isoladamente e em mistura com glyphosate $(0,42 \mathrm{~kg} / \mathrm{ha})$.

\section{CONCLUSÕES}

A aplicação do herbicida sulfentrazone em pré-emergência na cultura do milho, aplicado isoladamente nas doses de $0,15,0,30$ e $0,45 \mathrm{~kg} / \mathrm{ha}$ ou em mistura com acetochlor na dose de $2,52 \mathrm{~kg} / \mathrm{ha}$, apresenta seletividade e eficácia no controle das plantas daninhas Alternanthera tenella, Brachiaria plantaginea, Desmodium tortuosum, Acanthospermum hispidum, Bidens pilosa, Emilia sonchifolia, Portulaca oleracea e Blainvillea rhomboidea.

As plantas daninhas Alternanthera tenella e Commelina benghalensis são satisfatoriamente controladas pelo herbicida carfentrazone-ethyl aplicado isoladamente nas doses estudadas. As plantas daninhas Indigofera hirsuta, Brachiaria plantaginea, Desmodium tortuosum, Acanthospermum hispidum, Richardia brasiliensis, Sida rhombifolia e Portulaca oleracea são controladas satisfatoriamente apenas quando o herbicida carfentrazone-ethyl é aplicado em mistura com atrazine ou nicosulfuron.

O herbicida carfentrazone-ethyl nas doses 0,004 e 0,007 $\mathrm{kg} / \mathrm{ha}$ aplicado em pós-emergência na cultura do milho é seletivo e apresenta praticabilidade de uso isoladamente ou em mistura com o herbicida nicosulfuron, quando as plantas daninhas encontram-se no estádio de oito folhas verdadeiras ou em mistura com atrazine, quando as plantas daninhas encontram-se no estádio de quatro folhas verdadeiras.

\section{LITERATURA CITADA}

ALMEIDA, F.S. Eficácia de herbicidas em pós-emergência no controle de plantas daninhas na cultura do milho. In: Instituto Agronômico do Paraná. Plantio direto do Estado do Paraná. Londrina: IAPAR, 1981.p. 101-144 (Circular, 23).

BLACKSAW, R. E. HOE-39866 use in chemical fallow systems. Weed Technology, v.3, p.420-428, 1989a.

BLACKSAW, R.E. Synergistic mixes of DPX-A7881 and clopryralid in canola (Brassica napus). Weed Technology, v. 3, p.690-695, 1989 b.

COLBY, S.R. Calculating synergistic and antagonistic responses of herbicide combinations. Weeds, v.15, p.2022, 1967.

DUKE, S. O., LYDON, J.; BRCERRIL, J. M.; SHERMAN, L. P.; LEHNEN, L. P.; MATSUMOTO, H. Protoporphyrinogen oxidase-inhibiting herbicides. Weed Science, v. 39, p. 465-473, 1991.

FANCELLI, L. A.; DOURADO NETO, D. Manejo de plantas daninhas. In: FANCELLI, L.A.; DOURADO NETO, D., eds. Produção de milho. Guaíba: Agropecuária, 2000.p. 183-215.

HARKER, K. N.; O'SULLIVAN, P. A. Synergistic mixtures of sethoxydim and fluazifop on annual grass weeds. Weed Technology, v.5, p. 310-316, 1991.

HATZIOS, K.K. Synergistic interactions of tebuthiuron with EPTC + R-25788 and butylate + R-257788 in corn (Zea mays). Weed Science, v.29, p.601-604, 1989.

JORDAN, D. L.; YORK, A. C.; GRIFFIN, J. L.; CLAY, P. A.; VIDRINE, P. R.; REYNOLDS, E. D. B. Influence of application variables on efficacy of glyphosate. Weed Technology. v.11, p.354-362, 1997.

KUNERT, K. J; DODGE, A. D. Herbicide induced radical damage and antioxidative system. In: BOGER, P. \& SANDMANN, G. (eds). Target site of herbicide action, Boca Raton, Florida: CRC Press, 1989. p. 45-63. 1989.

LICH, J.M.; RENNER, K. A.; PENNER, D. Interaction of glyphosate with postemergence soybean (Glycine max) herbicides. Weed Science, v. 45, p. 12-21, 1997.

MINTON, B.W.; SHAW, E.R.; KURTZ, M.E. Postemergence grass and broadleaf herbicide interactions for red rice (Oryza sativa) control in soybeans (Glycine max). Weed Technology, v.3, p.329-334, 1989.

MONQUERO, P. A.; CHRISTOFFOLETI, P. J.; SANTOS, C. T. Glyphosate em mistura com herbicidas alternativos para o manejo das plantas daninhas. Planta Daninha, v.19, p.375-380, 2001.

RILEY, D.G.; SHAW, D.R. Influence of imazapyr on the control of pitted morningglory (Ipomoea lacunosa) and johnsongrass (Sorghum halepense) with chlorimuron, imazaquin, and imazethapyr. Weed Science, v.36, p.663666, 1988.

RILEY, D. G.; SHAW, D. R. Johnsongrass (Sorghum halepense) and pitted morningglory (Ipomoea lacunosa) control with imazaquin and imazethapyr. Weed Technology, v. 3, p. 95-98, 1989.

RODRIGUES, B.N.; LIMA, J.; YADA, I.F.V.; FORNAROLLI, D.A. Utilização de acetochlor e atrazine aplicados em mistura de tanque com dessecantes no sistema de plantio direto. Planta Daninha, v.18, p. 293-299, 2000. 
Manejo de plantas daninhas na cultura de milho com os herbicidas sulfentrazone e carfentrazone-ethyl

RODRIGUES, B. N.; ALMEIDA, F. S. Guia de herbicidas. Londrina: IAPAR, 1995.648p.

STARKE, R.J.; OLIVER, L.R. Interaction of glyphosate with chlorimuron, fomesafen, imazethapyr and sulfentrazone. Weed Science, v. 46, p.652-660, 1998.
SORENSON, V.M.; MEGGITT, W.F.; PENNER, D. The interaction of acifluorfen and bentazon in herbicidal combinations. Weed Science, v.35, p.449-456, 1987.

ZIMDHAL, R. L. Weed crop competition: a review. Corvallis: Oregon State University/International Plant Protection Center, 1990. 196p. 
